\title{
The Contingent Magnetic Variation in Migraine
}

\author{
G. Sartory, V. Gillessen, K. H. Grotemeyer, and T. Elbert* \\ Clinical Psychology, University of Wuppertal, \\ *Department of Psychology, University of Konstanz, Germany
}

Keywords: Contingent Magnetic Variation, migraine, dipole localization

\begin{abstract}
Migraine is characterized by an increased contingent negative variation. Separate comparisons of the early and late component showed that it was the former rather than the latter that was elevated. The function and localization of the early component are as yet poorly understood. The magneto-encephalogram was recorded in 16 patients with migraine and 17 healthy control subjects during a forewarned reaction time task. Neuromagnetic data were recorded with a 37-channel neuromagnetometer with the sensors being placed above the left hemisphere contralaterally to the side of the acoustic stimulation and the motor reaction. Subjects responded to the second of two low intensity sound stimuli that were administered with an interval of $4.5 \mathrm{~s}$. Migraine patients exhibited a significantly higher amplitude of the early component of the contingent magnetic variation (CMV) than controls. Groups did not differ with regard to the amplitude of the late component. Modeling the sources with a single moving equivalent current dipole (ECD) provided a high goodness of fit for the M100 (magnetic $\mathrm{N} 1$ ) in both groups and for the early component of the CMV in migraineurs but not controls. The activity of the early component was centered medially with respect to the M100-ECDs and more laterally so in migraine patients than in controls.
\end{abstract}

The contingent negative variation (CNV) is a negative potential shift which occurs in a forewarned reaction time task between the warning (S1) and imperative (S2) stimulus (Walter, Cooper, Aldridge, McCallum, \& Winter, 1964). Extending the interstimulus interval (ISI) between $\mathrm{S} 1$ and $\mathrm{S} 2$ to $3 \mathrm{~s}$ and more has revealed subcomponents of the CNV of which an early one peaks around $1 \mathrm{~s}$ and a late or "terminal" one peaks prior to S2 (see Rockstroh, Elbert, Canavan, Lutzenberger, \& Birbaumer 1989). The early component is most prominent at frontal and the late $\mathrm{CNV}$ at central electrode sites. The latter shows features similar to those of the Bereitschaftspotential (Kornhuber \& Deecke, 1964), i. e., it occurs preparatory to movement and its amplitude has been shown to vary inversely with subsequent reaction time (Rohrbaugh \& Gaillard, 1983).
CNV studies in clinical groups have almost invariably yielded decreased amplitudes (Sartory, 1985) with two notable exceptions, in obsessive-compulsive (Sartory \& Master, 1984) and in migraine patients (Boecker, TimsitBerthier, \& Brunia, 1990; Maertens de Noordhout, Timsit-Berthier, Timsit, \& Schoenen, 1986; Sartory, Besken, \& Pothmann, 1997; Schoenen, Maertens de Noordhout, Timsit-Berthier, \& Timsit, 1986; Timsit, Timsit-Berthier, Schoenen, \& Maertens de Noordhout, 1987). Schoenen et al. (1986) proposed that the elevated CNV amplitude in migraine was due to elevated central noradrenergic activity; CNV elevation could be shown to correlate with the clinical response to beta blockers. Kropp and Gerber (1995) found a slower habituation of the early CNV amplitude in migraine patients than controls and suggested that this was the cause of their elevated average. 
Analysis of the CNV components revealed a higher amplitude of the early rather than late segment in adult (Kropp \& Gerber, 1995) as well as childhood migraineurs (Sartory et al., 1997) compared with age-matched controls. There were no significant group differences with regard to the late component. Little is known of the functional role of the early component. It may reflect processes relating to the expectancy of S2, the imperative stimulus (van Boxtel, van de Boogart, \& Brunia, 1993), or an attentional or orienting response to S1 (Loveless, 1983). The latter position would be consistent with the finding of a retarded response habituation of the early wave over blocks of trials in migraineurs (Kropp \& Gerber, 1995). It may be indicative of their sustained attentional engagement not evident in healthy controls.

The investigation of cortical generators could shed light on the question of functional significance of slow potentials (Wikswo, Gevins, \& Williamson, 1993). Simultaneous magnetic and electric recordings of the auditory evoked response showed components of up to $50 \mathrm{~ms}$ to be located in the primary auditory cortex and $\mathrm{N} 1$ to result from activation of the secondary auditory areas (Pantev et al., 1995). Studies of the magnetic equivalent of the Bereitschaftspotential, recorded prior to spontaneous movement, found an initial slow-rising bilateral shift over frontal and central regions which is likely to be generated by the supplementary motor area (Deecke, Boschert, Brickett, \& Weinberg, 1985). Symmetric activation of both motor cortices was observed to start $600 \mathrm{~ms}$ and lateralized activity contralateral to movement 200-300 ms before movement onset (Cheyne, Weinberg, Gaetz, \& Jantzen, 1995). The Bereitschaftsfield culminated in the motor field and was followed by movement evoked fields up to $250 \mathrm{~ms}$ after movement onset (Nagamine, Kajola, Salmelin, Shibasaki, \& Hari, 1996). There is considerable intersubject variance with regard to the Bereitschaftsfield. Similar variability has been observed with regard to the late $\mathrm{CNV}$ component by Elbert, Rockstroh, Hamson, Pantev, and Hoke (1994) suggesting a distribution among motoric, sensory, and association areas in different subjects. The late CNV was impaired in neurological subjects with prefrontal cortex damage (Rosahl \& Knight, 1995). The selection of subjects on the basis of the strength and stationarity of their electrically recorded $\mathrm{CNV}$ resulted in one equivalent source contralateral to the side of movement close to the precentral cortex similar to the location found for the Bereitschaftspotential (Hultin et al., 1996). Ipsilateral equivalent sources were less consistent across subjects in this study.

The early component of the electrical CNV has been investigated less often with the MEG. A first study with healthy subjects yielded no consistent source owing to the high amount of intersubject variability (Elbert, et al.,
1994). Testing a single subject, Liu and colleagues (1996) noted, “. . . a long silent period between the warning and imperative stimuli is seen" in the averaged signal. Single trial analysis yielded some trials with sequences of activation in frontal and posterior areas. The latency of the activity differed between trials, hence the long silent periods when averaging them. As migraine patients tend to have a more pronounced early component of the electrical CNV (Sartory et al., 1997), it was thought the source localization might be more successful in these patients than in subjects with a variable early component. A MEG study of the CNV in migraine patients may therefore advance our understanding of processes relating to migraine and to the early CNV component at the same time.

The design of the present study was adapted from Proefrock and Hoke's (1995) study: A conventional forewarned reaction time task with soft tones and an S1-S2 interval of $4.5 \mathrm{~s}$ was administered to patients suffering from migraine and to healthy control subjects. The low intensity of the stimuli was thought to accentuate differences in sustained attention between the two groups. It has also been suggested that the CNV indicates cortical excitability (e. g., Elbert, 1993). The elevated amplitude in migraine patients may be related to improved S2 detection near the individual hearing threshold. This should result in an improved reaction time and error rate. The alternative assumption of increased attentional processes in migraine was further investigated with neuropsychological tests known to tap this function such as the digit span test, the digit symbol test, and trail making test (Reitan, 1958; Wechsler, 1955). The color word association test, a test of executive function, was added. It has been shown to be impaired in patients with compromised frontal lobe function (Linden et al., 1990). As the early component of the $\mathrm{CNV}$ is located fronto-centrally, it was of interest to find out whether or not migraine patients perform differently to controls in tasks of executive function. Given that obsessional-compulsive (OCD) patients showed a similarly elevated CNV amplitude as migraine patients (Sartory \& Master, 1984), subjects of the present study were also administered an OCD-inventory to assess whether or not migraine patients exhibited OCD features.

\section{Method}

Sixteen patients ( 15 women, mean age: 38 years, SD = 11.6) suffering from migraine according to the IHS Classification of Headaches (1988) and 17 healthy controls (all women, mean age: 27 years, $\mathrm{SD}=3.8$ ) took part in the study. Nine of the patients suffered from migraine 
with aura, six of them experienced no aura symptoms, and two suffered from menstrual migraine. None of the patients received prophylactic medication at the time of testing and none experienced a migraine attack during testing nor had they done so for at least three days. They also had not taken analgesic medication for at least three days. None of the controls took any medication or drugs. All subjects reported being right handed. Prior to taking part, subjects were given a detailed demonstration of the experimental procedure and apparatus and gave their informed consent apart from 2 additional control subjects who elected to withdraw from the laboratory procedure. One additional migraine patient terminated the laboratory session prematurely. Only her paper and pencil data were included in the data analysis. Controls received a small remuneration (DM 15.00 per hour of testing) to cover their travel expenses. This was taken care of by the health insurance of the patients.

\section{Apparatus}

Neuromagnetic data were recorded using a 37-channel neuromagnetometer (Magnes, Biomagnetic Technologies Inc., San Diego). The sensors are configured as firstorder axial gradiometers with a baseline of $50 \mathrm{~mm}$. The detection coils are arranged in an array of concentric circles with a diameter of $14.4 \mathrm{~cm}$ on a spherical surface. The coil diameter is $2.0 \mathrm{~cm}$ with a distance of $2.2 \mathrm{~cm}$ between the centers of two adjacent coils. Each gradiometer is connected to a Superconducting Quantum Interference Device (SQUID) which produces a voltage proportional to the magnetic field along the axis of the coil. Measurements were carried out in a magnetically shielded room. Acoustic stimuli were administered to the right ear via a non-magnetic and echo-free stimulus delivery system.

The Dewar was placed above the subject's left hemisphere, contralateral to the side of stimulation and movement. A sensor position indicator system determined the spatial locations of the sensors relative to the head. It also served to detect movement during recording. The sensor array was centered over a point lying $1.5 \mathrm{~cm}$ superior to the position T3 of the 10/20 system for electrode placement, as near as possible to the subject's head.

\section{Procedure}

Following the assessment of the individual auditory threshold, a forewarned reaction time task was administered. The warning stimulus (S1) consisted of a tone of $1000 \mathrm{~Hz}$, an intensity of $20 \mathrm{~dB}$ SL (SL: sensation level; i. e., relative to the individual's threshold for that tone), and lasted for $500 \mathrm{~ms}$. It was followed by the imperative stimulus (S2), a tone of $1000 \mathrm{~Hz}$ which lasted for $1000 \mathrm{~ms}$ unless it was switched off beforehand. It had an intensity of either 1,3 , or $5 \mathrm{~dB}$ SL. The sequence of intensities was randomized. There were no differences in the CMV between the three intensity levels and they were combined for analysis. The time delay between $\mathrm{S} 1$ onset and S2 onset was $4.5 \mathrm{~s}$. The intertrial interval was randomized between 9.5-12.5 s. Subjects were in a right lateral position with their head and neck supported by vacuum casts to prevent movement. They were instructed to place their thumb as quickly as possible upon the light of an optic fiber cable after hearing S2 in order to switch it off. Subjects were given practice trials until they mastered the procedure. Afterwards, recording started and trials were administered for $500 \mathrm{~s}$ followed by a short break allowing the transfer of data. At least 5 such blocks of trials were administered and more if the subject agreed. Five to seven blocks were in fact administered with a mean of 5.9 blocks. During the breaks between blocks, subjects were asked as to their well-being via intercom and told that they could move their legs briefly.

\section{Questionnaires and Tasks}

These were completed before the CMV recording.

- Trailmaking Test A and B (Reitan, 1958). Test A consists of 25 scattered numbers which have to be connected in ascending order. Test B consists of 13 scattered numbers and the letters $\mathrm{A}$ to $\mathrm{L}$ which have to be connected alternately. Both tests are timed. This test is considered an initial screening instrument of cognitive dysfunction.

- The Color Word Association Test (Linden et al., 1990) consists of 6 colored plates, each of which is associated with one of six words displayed on a card (e. g., red - opinion, green - soul, etc.). In the first run, subjects are asked to guess which word is associated with which color. Each guess is answered with "no" or "yes" by the examiner. In the following 5 runs, subjects are asked to remember the correct answer. The order in which the color cards are shown is different in each of the six runs as is the order of words in the word list. The number of erroneous answers is noted in each run and combined to a total error score. This test was designed to tap executive function; Linden et al. (1990) found that elderly Parkinson patients performed worse than age-matched controls while showing a similar performance in all other neuropsychological tests.

- Digit span (Wechsler, 1955). The subjects's immediate retention span is determined when repeating series of digits forward and backward. The measure is con- 
sidered to be an index of attention and short-term memory.

- Digit symbol test (Wechsler, 1955). Nine digits have to be substituted by symbols. The number of substitutions per minute constitute the score. This test has been shown to be sensitive to attentional impairment.

- Hamburg Obsessions and Compulsions Inventory (Zaworka, Hand, Jauernig, \& Lünenschloß, 1983). This self-rating scale consists of statements that are to be answered in terms of "applies" or "does not apply." The items are grouped into six subscales: A - checking, B - washing, C - tidying, D - counting, E - doubts, Fthoughts of injury, and also combine in a total score.

The administration of the neuropsychological tests and completion of the questionnaire took about an hour, after which subjects were allowed a short break before undergoing the laboratory procedure.

\section{Artifact Rejection and Reduction of MEG Data}

MEG data were sampled at a rate of $122.5 \mathrm{~Hz}$. Trials extended from $500 \mathrm{~ms}$ pre $\mathrm{S} 1$ onset (baseline) to $3500 \mathrm{~ms}$ post S2 onset; they were excluded from analysis if the difference between the maximum and minimum, computed across the whole $8.5 \mathrm{~s}$ epoch, exceeded 3.5 pico Tesla (pT) in any of the MEG channels. This criterion also served to exclude trials with large eye movements or blinks leading to elevated amplitudes in anterior channels. Subjects were excluded from further analysis if they had an artifact rate higher than $30 \%$. The artefact rejection procedure led to the elimination of 7 subjects ( 3 migraine patients and 4 controls) leaving 13 in each group for further analysis. In these remaining subjects, a mean of $12.3 \%$ trials were excluded, leaving 191 to be averaged.

Data were filtered using a low-pass of $20 \mathrm{~Hz}$ and baseline corrected. Root-mean-square (RMS) field values over the 37 recording channels were calculated for every sampling point. The following RMS data were extracted for further analysis: (1) M100: The maximum peak amplitude was determined within 50 to $200 \mathrm{~ms}$ post S1 onset and post S2 onset. The lengthy time window was necessary because of the low intensity of the tones which resulted in long peak latencies at $\mathrm{S} 2$. (2) CMV: RMS data were determined for each 0.24 -s epoch between 0.18 and $4.5 \mathrm{~s}$ post $\mathrm{S} 1$ onset resulting in $18 \mathrm{CMV}$ epochs. The RMS epochs were submitted to a principal component analysis. (3) Post-S2-PIMV (post imperative magnetic variation): The maximum RMS peak was determined within 350 and $800 \mathrm{~ms}$ post $\mathrm{S} 2$ onset.

\section{Dipole localization}

Source analyses using a single moving dipole model were applied to both CMV components. A spherical model was fitted to the digitized head shape of each subject and the location ( $\mathrm{x}, \mathrm{y}, \mathrm{z}$ positions), orientation, and amplitude of a best-fitting equivalent current dipole (Sarvas, 1987) were estimated for each data point in time. The origin of the head-based coordinate system determined by the sensor position indicator system - was the midpoint of the line between the preauricular points. This also constituted the lateral-medial y-axis with positive values towards the left preaucular point. The anterior-posterior $\mathrm{x}$-axis joined the $\mathrm{y}$-axis at the origin. The superior-inferior z-axis was perpendicular to the other two axes. Correlations between the theoretical field generated by the model and the observed field were calculated to estimate the goodness of fit of the model parameters. Only estimates with a goodness of fit above 0.90 and a confidence volume of less than $1000 \mathrm{~mm}^{3}$ were considered for further analysis. The $\mathrm{x}, \mathrm{y}, \mathrm{z}$ coordinates of the dipole location were calculated in the above described head frame-based Cartesian coordinate system.

\section{Results}

A representative example of the distribution of the early CMV component of a migraine patient is displayed in Figure 1. Group means of RMS data show that this component is less pronounced in healthy control subjects, as shown in Figure 2.

The principal component analysis (PCA) of RMS epochs and varimax rotation yielded three factors (Table 1) which accounted for $89.3 \%$ of the variance. The first factor $(64.4 \%)$ received the highest loadings from 2.6 to $4.5 \mathrm{~s}$ and can be considered as the late CMV component; the second factor $(16.7 \%$ ) showed the highest loadings from 1.4 to $2.6 \mathrm{~s}$ and represents a middle component of the CMV; finally, the third factor $(8.2 \%)$ combined loadings from 0.24 to $1.4 \mathrm{~s}$ and is therefore the early CMV component. The small \% variance accounted for by this factor is a reflection of the short duration of the early component.

Factor scores were extracted for each subject whereby individual amplitude scores are weighted by the factor loading and integrated. Groups were compared with regard to the three CMV components. Migraine patients showed a significantly higher early CMV than controls (Student's $t=2.07, \mathrm{df}=24, P<.05$ ). Neither the late nor the middle CMV component yielded significant group differences.

\section{Source Localization}

Using the source localization procedure and evaluation criteria described in the methods section, the equivalent 


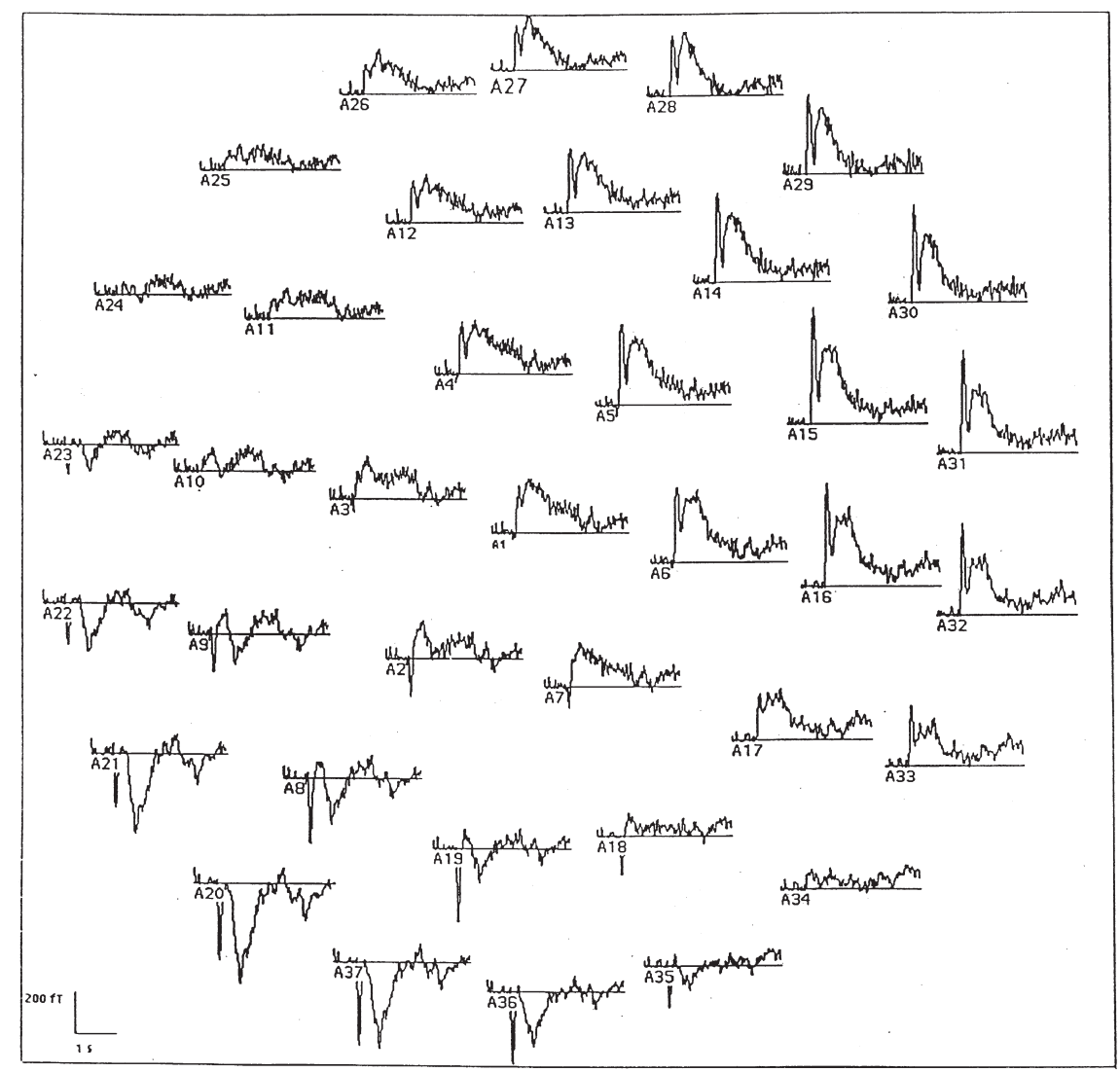

Figure 1 Representative example of the CMV of a migraine patient. The Dewar was placed above the left side of the head. The sensor position A1 corresponds to the electrode placement of $\mathrm{T} 3$ with the vertex at the top. The initial $485 \mathrm{~ms}$ constitute the baseline followed by an epoch of $2.8 \mathrm{~s}$. Given the temporal placement of the Dewar, the latter part of the trial containing the motor response is not displayed.

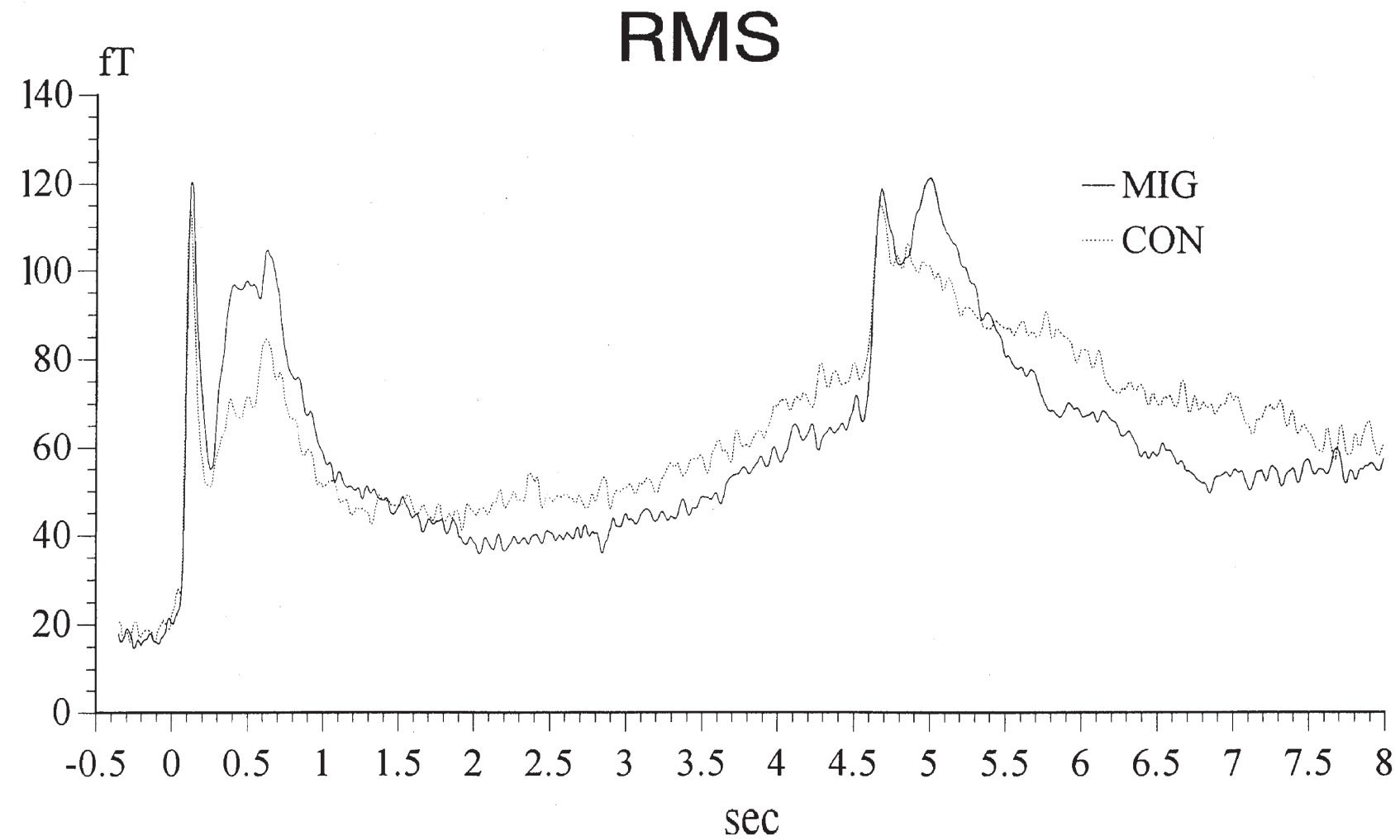

Figure 2 Group means of the strength of the magnetic field in femtotesla (fT), expressed as root mean square (RMS) over the 37 recording channels, in migraine patients (MIG; $N=13$ ) and healthy controls (CON; $N=13$ ) during a forewarned reaction time task. The $\mathrm{S} 1-\mathrm{S} 2$ interval is $4.5 \mathrm{~s}$. Groups differed only with regard to the early CMV component. The group mean of the factor score was .38 (SD = 1.13) in migraineurs and $-.38(S D=.71)$ in controls. 


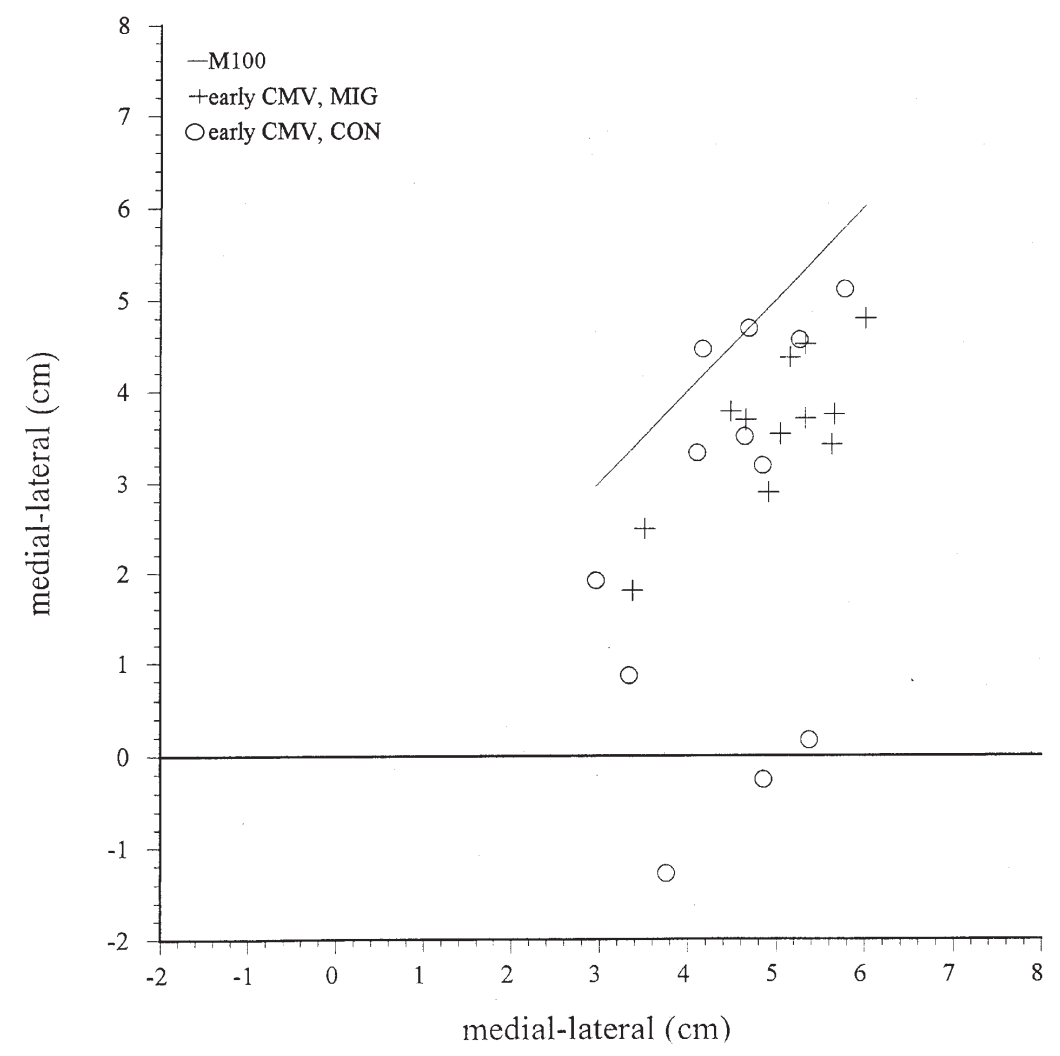

Figure 3 Equivalent current dipole coordinates of the early CMV component in migraine patients (MIG) and healthy controls (CON). Coordinates are displayed in the medial-lateral axis relative to the position of the M100; the higher the values the more lateral the spatial position.

Table 1 Principal component analysis and Varimax rotation of the 0.24 s CMV-epochs of all subjects.

\begin{tabular}{rlll}
\hline Epochs (ms) & $\begin{array}{l}\text { Factor 1 } \\
\text { late CMV }\end{array}$ & $\begin{array}{l}\text { Factor 2 } \\
\text { middle CMV }\end{array}$ & $\begin{array}{l}\text { Factor 3 } \\
\text { early CMV }\end{array}$ \\
\hline $244.9-481.6$ & - & - & .873 \\
$481.6-718.4$ & - & - & .924 \\
$718.4-955.1$ & - & - & .940 \\
$955.1-1191.8$ & - & - & .886 \\
$1191.8-1428.6$ & - & .606 & .691 \\
$1428.6-1665.3$ & - & .816 & - \\
$1665.3-1902.0$ & - & .767 & - \\
$1902.0-1238.8$ & - & .823 & - \\
$1238.8-2375.5$ & - & .828 & - \\
$2375.5-2612.2$ & .526 & .781 & - \\
$2612.2-2849.0$ & .693 & .584 & - \\
$2849.0-3085.7$ & .811 & - & - \\
$3085.7-3322.4$ & .906 & - & - \\
$3322.4-3559.1$ & .916 & - & - \\
$3559.1-3795.1$ & .891 & - & - \\
$3795.1-4032.7$ & .872 & - & - \\
$4032.7-4269.4$ & .834 & - & - \\
$4269.4-4506.1$ & .789 & - & - \\
\hline \% Variance & 64.4 & 16.7 & 8.22 \\
\hline
\end{tabular}

source locations for M100 and for the early CMV were determined for each subject. This led to the exclusion of two more subjects who failed to satisfy criteria for the presence of a single equivalent current dipole, leaving 12 in each group. The equivalent current dipole location of
Table 2 Group means (SDs) of the coordinates of the equivalent current dipole locations of the M100 and the early CMV in migraine patients $(N=12)$ and healthy controls $(N=12)$. Data are presented in $\mathrm{cm}$.

\begin{tabular}{lrr}
\hline & \multicolumn{1}{c}{ Migraine } & \multicolumn{1}{c}{ Controls } \\
\hline Posterior-Anterior M100 & $.89(1.35)$ & $.54(.99)$ \\
$\quad$ early CMV & $.70(1.28)$ & $.73(1.70)$ \\
Medial-Lateral M100 & $4.94(.81)$ & $4.49(.84)$ \\
$\quad$ early CMV & $3.56(.85)$ & $2.29(2.09)$ \\
Inferior-Superior M100 & $5.22(1.14)$ & $5.24(.98)$ \\
$\quad$ early CMV & $4.89(1.28)$ & $5.24(1.26)$ \\
\hline
\end{tabular}

the M100 was used as the internal marker of the location of the subject's auditory cortex (Pantev, Eulitz, Elbert, \& Hoke, 1994) and was employed as a point in space to which early CMV was referred. Table 2 shows group means and SDs of the coordinates of the equivalent current dipoles of the M100 and early CMV.

Analyses of variance were carried out separately for coordinates of the three axes (medial-lateral, anteriorposterior, and superior-inferior) with a GROUP $(2) \times$ LATENCY (2) design, whereby the latter constituted a withingroup factor comparing M100 and CMV coordinates. Only the medial-lateral axis yielded significant effects (Figure 3). The equivalent current dipole of the early CMV is more medially situated than that of the M100 $(F(1,22)=34.57, P<.01)$. Groups also differed marginally $(F(1,22)=4.14, P<.06)$ with the migraine group 
showing a more laterally situated equivalent current dipole of the early CMV than controls. The latter also exhibited a markedly greater SD with regard to this measure than migraine patients.

\section{Motor Reaction}

Groups did not differ significantly with regard to reaction time (migraineurs: $576 \mathrm{~ms}$, controls: $553 \mathrm{~ms}$ ) or error rate, i. e., number of erroneous button presses (migraineurs: 3.0, controls: 3.3 ).

\section{Neuropsychological Tests}

Group means and SDs of neuropsychological tests and of the total score of the Hamburg OC- Inventory are displayed in Table 3. None of the neuropsychological tests yielded significant group differences nor did the total OCD score. Two of the six subscales showed significant effects. Migraine patients indicated a slightly greater concern about washing habits (subscale B) than controls $(\mathrm{M}=5.7, \mathrm{SD}=2.9$ and $\mathrm{M}=3.4, \mathrm{SD}=2.4$, respectively; $P<.05$ ); the latter were, however, more concerned about injury obsessions (subscale $\mathrm{F}$ ) than patients $(\mathrm{M}=2.3, \mathrm{SD}=2.4$ and $\mathrm{M}=0.7, \mathrm{SD}=0.9$, respectively; $P<.05)$. None of the subjects showed scores within the clinical range.

Table 3 Group means of neuropsychological tests and the Hamburg obsessions and compulsions inventory.

\begin{tabular}{lrr}
\hline & \multicolumn{1}{l}{ Migraine } & \multicolumn{1}{l}{ Controls } \\
\hline Trailmaking-Test A (s) & $26.22(11.64)$ & $21.67(7.03)$ \\
Trailmaking-Test B (s) & $66.15(29.03)$ & $51.81(16.78)$ \\
Digit span (forward) & $6.31(1.18)$ & $6.31(0.79)$ \\
Digit span (backward) & $5.15(1.28)$ & $5.63(1.46)$ \\
Digit symbol test & $36.77(8.33)$ & $40.18(6.77)$ \\
Color word association test & $33.69(19.41)$ & $32.07(15.95)$ \\
Hamburg obsessions \&t & & \\
compulsions inventory & $33.60(16.84)$ & $33.25(11.95)$ \\
\hline
\end{tabular}

\section{Discussion}

The early component of the CMV, expressed as signal power (RMS) across the 37 channels, showed a higher amplitude in migraine patients than controls, whereas none of the other components differed between groups. The finding of an elevated CMV is consistent with previous reports of an overall higher $\mathrm{CNV}$ amplitude in migraine patients than healthy controls (Kropp \& Gerber, 1995; Maertens de Noordhout et al., 1986; Sartory et al., 1997; Schoenen, et al., 1986; Timsit et al., 1987).
In a separate analysis of the early and late component, Sartory et al. (1997) also found only the early component to be elevated in children with migraine. The principal component analysis of the CMV, used here as a means of reducing data for further analysis, resulted in similar components to those usually found in the electric $\mathrm{CNV}$ - an early, middle, and late component.

Results of the source localization of the early component differed between groups. The estimated source location was found to be more laterally situated in migraine patients than in controls. There were no group differences with regard to the other two coordinates of that component. It could be argued that the more lateral location in migraine patients also produced the stronger signal power at that latency. Healthy control subjects failed to yield a consistent area of activation with regard to the early CNV component as has been shown before (Elbert et al., 1994). A number of reasons could account for the equivalent current dipole's failure to produce a valid source location. The location of ECD sources in control subjects might be better explained by assuming the existence of a configuration of multiple and/or distributed sources (Mosher, Spencer, Leahy, \& Lewis, 1993). Liu et al. (1996) found multiple areas of activation even at $100 \mathrm{~ms}$ after S1, albeit in a single subject. The averaging procedure may have eliminated components that are not time locked to $\mathrm{S} 1$ or else are only evident in some trials. Finally, sources may not have been detected due to the limited sensor positioning. Given all these limitations, results nonetheless indicate group differences in the structure and location of the generators. Migraine patients have a more consistent focal activation than healthy controls which may, in turn, give rise to the former group's frequently observed elevated early $\mathrm{CNV}$ component. It is as yet unclear which aspect of the migraine pathology should give rise to this localized generator during a forewarned reaction time task.

Migraine patients were older (38 years) than controls (27 years) and it could be argued that the age difference accounted for the group differences in the early CMV. Re-analysis of data from healthy subjects of an earlier study (Elbert et al., 1994) yielded a non significant, negative correlation of $r=-.32$ between age and RMS of the early CMV component. There is thus no marked change in this component with age. Had age affected the present results, migraine patients - being older than controls should have produced a diminished early CMV component which was, however, not the case.

Estimated source location of the M100 to tones of different frequencies is well established (Pantev et al., 1994). It is known to be localized in the auditory cortex and the coordinates found in the present study are similar to those published previously (Pantev et al., 1994). They are identical for both groups. These coordinates can 
therefore conveniently serve as reference points for the estimated source location of other components. The ECD coordinates of the early CMV in migraine patients are consistent with Boecker and Brunia's (1994) finding of the insula as location of the dipole generating the expectancy component of the stimulus preceding negativity (SPN). Little is known of the functional role of this area of the brain. Moreover, it cannot be excluded that the generator resides in the nearby pallidum. MRI analysis could have rendered the localization more precise but was not available.

The results are consistent with Kropp and Gerber's (1995) suggestion that the early CNV is subject to habituation in healthy subjects which is reduced or absent in migraineurs. Given the large number of trials necessary to assess the magnetic field, any habituating component would soon be submerged in the averaging process. The diminishing strength of the temporal source may thereby have produced the inconsistent results regarding the source localization in controls. By contrast, the migraineurs may have continually activated the temporal source providing a consistent temporal generator. It remains, however, unclear which particular functional or clinical feature is related to this result. Habituation of the early component implies that it represents an orienting response. There are so far no other data suggesting that migraine is associated with an elevated orienting response. In the present study, according to neuropsychological tests and RT, migraine patients could not be shown to be characterized by elevated sustained attention, nor did they exhibit obsessional features which may have been interpreted as over-attending to irrelevant details.

\section{Acknowledgment}

The study was carried out at the Center of Biomagnetism/Experimental Audiology of the University of Münster during Dr. Elbert's employment there.

\section{References}

Boecker, K.B.E., \& Brunia, C.H.M. (1994). Spatiotemporal dipole modeling of the stimulus preceding negativity (SPN) prior to knowledge of results. Psychophysiology, 31, 27-28.

Boecker, K.B.E., Timsit-Berthier, M., \& Brunia, C.H.M. (1990). Contingent negative variation in migraine. Headache, 30, 604609.

Cheyne, D., Weinberg, H., Gaetz, W., \& Jantzen, K.J. (1995). Motor cortex activity and predicting side of movement: Neural network and dipole analysis of pre-movement magnetic fields. Neuroscience Letters, 188, 81-84.
Deecke, L., Boschert, J., Weinberg, H., \& Brickett, P. (1983). Magnetic fields of the human brain preceding voluntary foot and toe movements. Experimental Brain Research, 52, 81-86.

Elbert, T. (1993). Slow cortical potentials reflect the regulation of cortical excitability. In W.C. McCallum \& S.H. Curry (Eds.), Slow potential changes in the human brain (pp. 235-251). New York: Plenum Press.

Elbert, T., Rockstroh, B., Hampson, S., Pantev, C., \& Hoke, M. (1994). The magnetic counterpart of the contingent negative variation. Electroencephalography \& Clinical Neurophysiology, 92, 262-272.

Hultin, L., Rossini, P., Romani, G. L., Hogstedt, P., Tecchio, F., \& Przella, A. (1996). Neuromagnetic localization of the late component of the contingent negative variation. Electroencephalography \& Clinical Neurophysiology, 98, 435-448.

International Headache Society Headache Classification Committee (1988). Classification and diagnostic criteria for headache disorders, cranial neuralgias and facial pain. Cephalalgia, 8 (Suppl. 7), 1-96.

Kornhuber, H.H., \& Deecke, L. (1964). Hirnpotentialveränderungen beim Menschen vor und nach Willkürbewegungen, dargestellt mit Magnetbandspeicherung und Rückwärtsanalyse. Pflügers Archiv Gesamte Physiologie, 281, 52.

Kropp, P., \& Gerber, W. D. (1995). Contingent negative variation during migraine attack and interval: Evidence of normalization of slow potentials during the attack. Cephalalgia, 15, 123-128.

Linden, A., Bracke-Tolkmitt, R., Lutzenberger, W., Canavan, A.G.M., Scholz, E., Diener, H.-C., \& Birbaumer, N. (1990). Slow cortical potentials in Parkinsonian patients during the course of an associative learning task. Journal of Psychophysiology, 5, 145-162.

Liu, M.J., Fenwick, P.B.C., Lumsden, J., Lever, C., Stephan, K.M., \& Ioannides, A.A. (1996). Averaged and single-trial analysis of cortical activation sequences in movement preparation, initiation, and inhibition. Human Brain Mapping, 4, 254-264.

Loveless, N. (1983). The orienting response and evoked potentials in man. In D. Siddle (Ed.), Orienting and habituation: Perspectives in human research (pp. 71-108). Chichester: John Wiley.

Maertens de Noordhout, A., Timsit-Berthier, M., Timsit, M., \& Schoenen, J. (1986). Contingent negative variation in headache. Annals of Neurology, 19, 78-80.

Mosher, J.C., Spencer, M.E., Leahy, R.M., \& Lewis, P.S. (1993). Error bounds for EEG and MEG dipole source localization. Electroencephalography \& Clinical Neurophysiology, 86, 303-321.

Nagamine, T., Kajola, M., Salmelin, R., Shibasaki, H., \& Hari, R. (1996). Movement-related slow cortical magnetic fields and changes of spontaneous MEG- and EEG-brain rhythms. Electroencephalography \& Clinical Neurophysiology, 99, 274286.

Pantev, C., Bertrand, O., Eulitz, C., Verkindt, C., Hampson, S., Schuierer, G., \& Elbert, T. (1995). Specific tonotopic organizations of different areas of the human auditory cortex revealed by simultaneous magnetic and electric recordings. Electroencephalography \& Clinical Neurophysiology, 94, 26-40.

Pantev, C., Eulitz, C., Elbert, T., \& Hoke, M. (1994). The auditory evoked sustained field: Origin and frequency dependence. Electroencephalography \& Clinical Neurophysiology, 90, 8290.

Proefrock, E.S., \& Hoke, M. (1995). Contingent magnetic variation (CMV) studied with stimuli close to the hearing threshold 
in normal subjects and tinnitus patients. In C. Baumgartner et al. (Eds.), Biomagnetism: Fundamental research and clinical applications (pp. 234-239). Amsterdam: Elsevier, IOS Press.

Reitan, R.M. (1958). Validity of the Trailmaking Test as an indication of organic brain damage. Perceptual Motor Skills, 8, 271-276.

Rockstroh, B., Elbert, T., Canavan, A.G.M., Lutzenberger, W., \& Birbaumer, N. (1989). Slow cortical potentials and behaviour. Munich: Urban and Schwarzenberg.

Rohrbaugh, J.W., \& Gaillard, A.W.K. (1983). Sensory and motor aspects of the Contingent Negative Variation. In A.W.K. Gaillard \& W. Ritter (Eds.), Tutorials in ERP-research: Endogenous components (pp. 269-310). Amsterdam: North Holland.

Rosahl, S.K., \& Knight, R.T. (1995). Role of prefrontal cortex in generation of the contingent negative variation. Cerebral Cortex, 5, 123-134.

Sartory, G. (1985). The CNV in psychiatric states. In D. Papakostopoulos, S. Butler, \& I. Martin (Eds.), Clinical and experimental neuropsychophysiology. London: Croom Helm.

Sartory, G., Besken, E., \& Pothmann, R. (1997). The contingent negative variation in childhood migraine. Journal of Psychophysiology, 11, 138-146.

Sartory, G., \& Master, D. (1984). Contingent negative variation in obsessional compulsive patients. Biological Psychology, 18, 253-267.

Sarvas, J. (1987). Basic mathematical and electromagnetic concepts of the biomagnetic inverse problem. Physical \& Medical Biology, 32, 11-22.

Schoenen, J., Maertens de Noordhout, A., Timsit-Berthier, M., \& Timsit, M. (1986). Contingent negative variation and efficacy of beta-blocking agents in migraine. Cephalalgia, 6, 229-233.
Timsit, M., Timsit-Berthier, M., Schoenen, J., \& Maertens de Noordhout, A. (1987). Intérèt de l'étude de la VCN dans les migraines et céphalées de tension. Neurophysiologie Clinique, 17, 259-270.

Van Boxtel, A., Van de Boogart, B., \& Brunia, C. M. (1993). The contingent negative variation in a choice reaction time task. Psychophysiology, 30, 11-23.

Walter, W.G., Cooper, R., Aldridge, V.J., McCallum, W.C., \& Winter, A.L. (1964). Contingent negative variation: An electric sign of sensorimotor association and expectancy in the human brain. Nature, 203, 380-384.

Wechsler, D. (1955). Manual for the Wechsler Adult Intelligence Scale. New York: Psychological Corporation.

Wikswo, J.P., Gevins, A., \& Williamson, S.J. (1993) The future of the EEG and MEG. Electroencephalography \& Clinical Neurophysiology, 87, 1-9.

Zaworka, W., Hand, I., Jauernig, G., \& Lünenschloß, K. (1983) Hamburger Zwangsinventar, HZI. Weinheim: Beltz.

\author{
Gudrun Sartory \\ Clinical Psychology, FB.3 \\ University of Wuppertal \\ Max-Horkheimer-Strasse 20 \\ D-42097 Wuppertal \\ Germany \\ Tel. +49 202 439-2722 \\ Fax +49 202 439-2824 \\ E-mail Sartory@uni-wuppertal.de
}

